\title{
ULTRA-THIN $\mathrm{P}^{++}$MONOCRYSTALLINE SILICON MICROSTRUCTURES
}

\author{
Chunchieh Huang and Khalil Najafi \\ Center for Integrated Sensors and Circuits, University of Michigan \\ 1301 Beal Avenue, Ann Arbor, Michigan 48109-2122
}

\begin{abstract}
This paper discusses the fabrication of submicron $\mathrm{p}^{++}$ microstructures for a number of MEMS applications using boron ion implantation, rapid thermal annealing, and boron etch-stop. To form these thin structures, the silicon is implanted with boron at an energy of $40 \mathrm{keV}$ and doses of $5 \times 10^{15} \mathrm{~cm}^{-2}$ and $7 \times 10^{15} \mathrm{~cm}^{2}$, which produce a peak concentration of more than $10^{20} \mathrm{~cm}^{-3}$, sufficient for achieving an effective etch-stop in EDP. The thickness of the $\mathrm{p}^{++}$ layer varies from 0.2 to $0.3 \mu \mathrm{m}$ depending on the annealing time and temperature. A number of microstructures, including thin silicon diaphragms as large as $2 \mathrm{~mm}$ on a side and $0.2 \mu \mathrm{m}$ thick, hot wire anemometers with a TCR of $\sim 1600 \mathrm{ppm} /{ }^{\circ} \mathrm{C}$, and piezoresistive sound detectors, have been fabricated with high reproducibility, uniformity, and yield.
\end{abstract}

\section{INTRODUCTION}

Operation of many physical sensors is based on thin membranes or beams which deflect in response to an external parameter. Generally, the sensitivity of the sensor is strongly dependent on the thickness of these microstructures. One common technique for fabricating thin microstructures is based on hightemperature boron diffusion and boron etch-stop [1]. Forming microstructures from $\mathrm{p}^{++}$silicon has been a powerful technique for fabricating a variety of high-performance sensors [2]. This technology offers several features, including the ability to form single-crystalline silicon microstructures which possess reliable and reproducible material properties, process simplicity, and reproducibility. However, thermal diffusion limits the minimum thickness of $\mathrm{a} \mathrm{p}^{++}$film to $>2-3 \mu \mathrm{m}$ due to the high temperature process which is necessary to create a high boron concentration $\left(>9 \times 10^{19} \mathrm{~cm}^{-3}\right)$ for achieving an effective etch-stop. The ability to form submicron $\mathrm{p}^{++}$membranes is instrumental to many emerging applications which require a wide dynamic range and high sensitivity. This paper presents the fabrication of submicron $\mathrm{p}^{\text {t+ }}$ microstructures using boron ion implantation and EDP etching. Extensive simulation and experimental results are presented to define the limits and capabilities of this process, and examples of various devices fabricated using this technology are discussed.

\section{FABRICATION PROCESS}

The most critical step in the fabrication of submicron $\mathrm{p}^{++}$ silicon microstructures is determining the most appropriate implant and annealing parameters, and choosing an appropriate etchant for achieving a reliable and uniform etch-stop. The etch-stop characteristics of boron-doped silicon in $\mathrm{KOH}$ and EDP as a function of boron concentration have been studied and characterized by other researchers [3]. In order to achieve an effective etch-stop, the ratio of the etch rates between undoped and highly-doped silicon should be generally higher than 100 . As a result, for an effective boron etch-stop in EDP, the boron concentration has to be $>9 \times 10^{19} \mathrm{~cm}^{3}$. In order to obtain a thin microstructure, this region of high concentration has to be confined to a narrow region, which is possible using ion implantation.
SUPREM simulation has been used to determine the range of implantation energies, doses, and annealing steps. Table I shows the simulated thickness of a $\mathrm{p}^{++}$layer for various implant conditions (annealed at $1100^{\circ} \mathrm{C}$ for $30 \mathrm{sec}$.). As can be seen, the thickness varies from $0.3 \mu \mathrm{m}$ to $0.5 \mu \mathrm{m}$ by changing the dose and the energy; higher energies and doses produce thicker layers. Since the final thickness is a strong function of boron concentration, it can also be altered by either implanting the boron through an oxide mask, or by post-implant annealing under different conditions. Figure 1 shows the simulated boron concentration profiles for four different annealing temperatures and times after an implantation dose of $7 \times 10^{15} \mathrm{~cm}^{-2}$ and energy of $40 \mathrm{keV}$. The maximum doping concentration is about $2.1 \times 10^{20} \mathrm{~cm}^{3}$ after rapid thermal annealing (RTA) at $1100^{\circ} \mathrm{C}$ for $30 \mathrm{sec}$. Such a high concentration is sufficient for achieving an effective etch-stop.

The determination of the most suitable annealing step is dependent on the device structure. As can be seen form Figure 1, the surface concentration of the implanted silicon after a $1000^{\circ} \mathrm{C}$ anneal is much lower than that after an $1100^{\circ} \mathrm{C}$ anneal. Therefore, if the ion implantation layer is protected from EDP from the front side, then either of these annealing steps can be used depending on the required thickness. Otherwise, an $1100^{\circ} \mathrm{C}$ anneal should be used to produce a high boron concentration for an effective etchstop. As will be presented later, these simulated results nicely match our experimental results.

Another important and critical element in the formation of thin $\mathrm{p}^{++}$layers is the silicon etchant used for dissolving the undoped layer and stopping on the $\mathrm{p}^{++}$film. Typically two wet etchants are used in bulk silicon micromachining, $\mathrm{KOH}$ and EDP. Although Folkmer et al. did successfully fabricate $\mathrm{p}^{++}$silicon structures using $\mathrm{KOH}$ [4], the temperature and concentration of the $\mathrm{KOH}$ bath needed to be well controlled to prevent $\mathrm{p}^{++}$silicon overetching. This is primarily because $\mathrm{KOH}$ has a lower etch-stop selectivity than EDP [3]. In the experiments reported here we have used EDP as the silicon etchant with a composition of: $150 \mathrm{ml}$ ethylenediamine, $48 \mathrm{~g}$ catechol, $48 \mathrm{ml}$ water, and $0.9 \mathrm{~g}$ pyrazine. The EDP temperature is $104^{\circ} \mathrm{C}$ which provides a better etch rate control. The total EDP time is between $90 \mathrm{~min}$. up to three hours. For an EDP etch at a higher temperature or much longer period than described above, the thin implanted $\mathrm{p}^{++}$layer could be attacked by EDP. Although the surface concentration of the implanted silicon wafers after anneal is higher than $9 \times 10^{19} \mathrm{~cm}^{-3}$, there is still a finite etch rate for the implanted region. From [3], the ratio of the etch rates in EDP between undoped and highly-doped silicon $\left(C_{B}=2.1 \times 10^{20} \mathrm{~cm}^{-3}\right)$ is about $1: 4 \times 10^{-4}$. As a result, the EDP etch will consume $\sim 800 \AA$ of the highly-doped silicon in the same time it will take EDP to dissolve away $200 \mu \mathrm{m}$ of undoped silicon (this assumes that the $\mathrm{p}^{++}$silicon is not protected from the EDP during this etch). The silicon sample has to be removed from EDP as soon as the undoped silicon is etched away to prevent overetching the implanted layer.

Determining the most appropriate implant energy and dose is dependent on the particular application and the corresponding fabrication sequences. On the one hand, because of the finite etch 
rate of highly-doped silicon in EDP, a low-energy, low-dose implant is not favorable. On the other hand, to fabricate extremely thin structures and to reduce production costs, a high-energy, highdose implant is not desirable. As indicated by the data in Table I, the designer has fairly wide latitude in choosing the best energy and dose to suit a particular application. For all of the devices reported in this paper, we used an implant energy of $40 \mathrm{keV}$, and doses of $5 \times 10^{15} \mathrm{~cm}^{-2}$ and $7 \times 10^{15} \mathrm{~cm}^{-2}$.

Table I: SUPREM simulation results

\begin{tabular}{|c|c|c|}
\hline Dose $\left(\mathrm{cm}^{-2}\right)$ & Energy $(\mathrm{keV})$ & Etch-stop $(\mu \mathrm{m})$ \\
\hline $5 \times 10^{15}$ & 30 & 0.28 \\
\hline $5 \times 10^{15}$ & 40 & 0.31 \\
\hline $5 \times 10^{15}$ & 50 & 0.33 \\
\hline $5 \times 10^{15}$ & 60 & 0.36 \\
\hline $7 \times 10^{15}$ & 30 & 0.33 \\
\hline $7 \times 10^{15}$ & 40 & 0.36 \\
\hline $7 \times 10^{15}$ & 50 & 0.38 \\
\hline $7 \times 10^{15}$ & 60 & 0.41 \\
\hline $1 \times 10^{16}$ & 30 & 0.37 \\
\hline $1 \times 10^{16}$ & 40 & 0.40 \\
\hline $1 \times 10^{16}$ & 50 & 0.42 \\
\hline $1 \times 10^{16}$ & 60 & 0.45 \\
\hline
\end{tabular}

* Etch-stop layer thickness is based on the silicon thickness at a boron concentration of $9 \times 10^{19} \mathrm{~cm}^{-3}$.

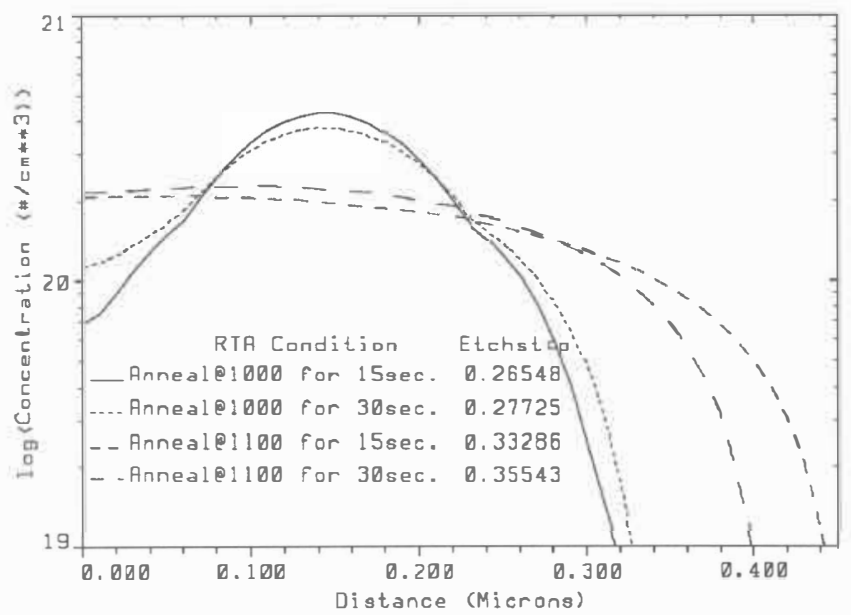

Figure 1: Boron concentration profile after various annealing temperatures and times.

\section{EXAMPLES}

The above technology has been applied to fabricate a number of devices, including thin silicon membranes for capacitive pressure sensors, $\mathrm{p}^{++}$silicon wires for flow sensors, and $\mathrm{p}^{++}$resistors for piezoresistive pressure sensors and sound detectors. Thin membrane fabrication is performed in standard bulk micromachining, while the silicon wires and piezoresistive sensors are fabricated using the dissolved wafer process [5]. The fabrication of each of these devices will be briefly described below.

To fabricate the ultra-thin silicon membranes, the front side of a silicon wafer was first implanted with boron at $40 \mathrm{keV}$ at a dose of $7 \times 10^{15} \mathrm{~cm}^{-2}$. After implantation, the wafer is thinned down to $200 \mu \mathrm{m}$ to reduce the EDP etch time. Note that EDP still etches highly-doped silicon at a slow rate because the front side of the wafer is exposed to the EDP etch during the entire etch period. Next, the backside of the silicon wafer is covered with a $2000 \AA$ layer of PECVD nitride, which is deposited at a temperature of $400^{\circ} \mathrm{C}$ for 10 minutes. Low temperature dielectric deposition is important for obtaining thin $\mathrm{p}^{\text {t+ }}$ layers because high temperature processes will drive the boron deeper into the silicon substrate and lower the dopant concentration. The PECVD dielectric layer is now patterned to create windows for the EDP etch, which etches through the entire thickness of the wafer and stops on the $\mathrm{p}^{++}$film. Figure 2 shows a silicon membrane formed using EDP etching following an RTA at $1000^{\circ} \mathrm{C}$ for $20 \mathrm{sec}$. The diaphragm is buckled due to non-uniform stress distribution. This is easily corrected by performing a second RTA at $1100^{\circ} \mathrm{C}$ for $30 \mathrm{sec}$. after the EDP etch, as shown in Figure 3. The diaphragm thickness is about $\sim 2000 \AA$ which is very close to the simulation result taking the finite etch rate of highly-doped silicon into consideration.

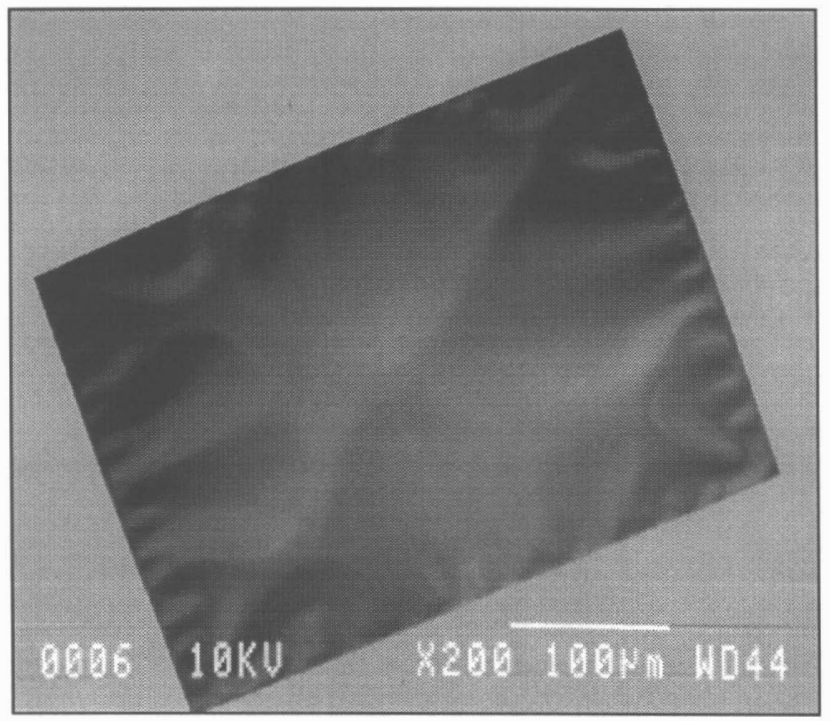

Figure 2: Thin $p^{++}$silicon membrane after 3 hours EDP etch. The membrane is buckled after release.

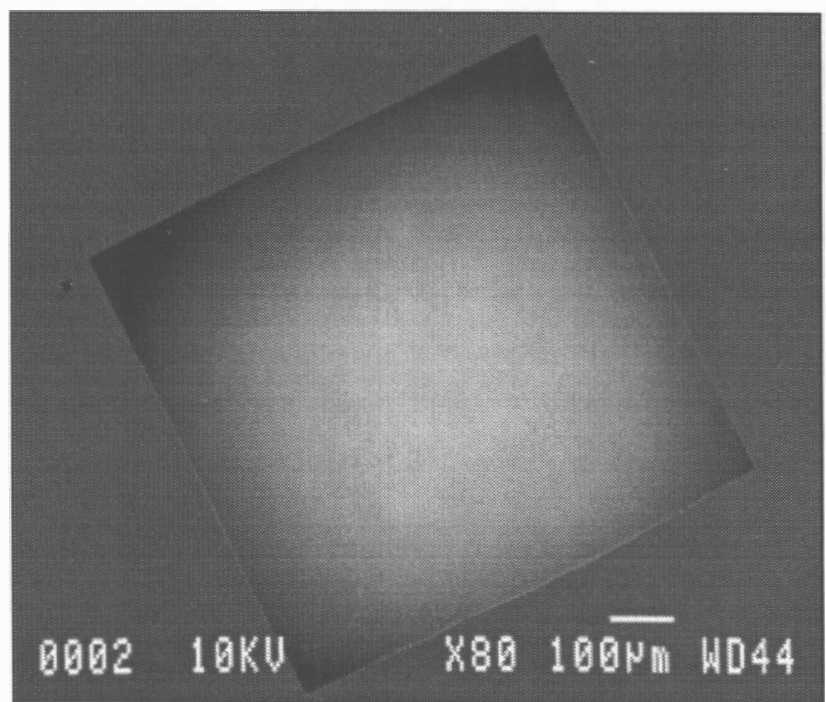

Figure 3: The $800 \mu m$-square silicon membrane is flat after RTA at $1100^{\circ} \mathrm{C}$ for $30 \mathrm{sec}$.

In order to obtain a flat diaphragm, one can also perform the RTA anneal at $1100^{\circ} \mathrm{C}$ before EDP. Note that since the anneal time is very short and since the anneal is performed using an RTA, one can easily perform the annealing step after the diaphragms are released since there is no need for any additional wet processing or 
cleaning of the wafer. The above diaphragms can be uniformly and reliably fabricated. Thickness uniformity is mainly dependent on the temperature uniformity of the EDP etch bath. In all of our experiments we etched only pieces of a 4" wafer, but in all of these experiments we obtained intact and flat diaphragms ranging in size from $300 \mu \mathrm{m}$-square to $2 \mathrm{~mm} \times 4 \mathrm{~mm}$. Figure 4 shows the cross section and a close-up view of one of these ultra-thin membranes.

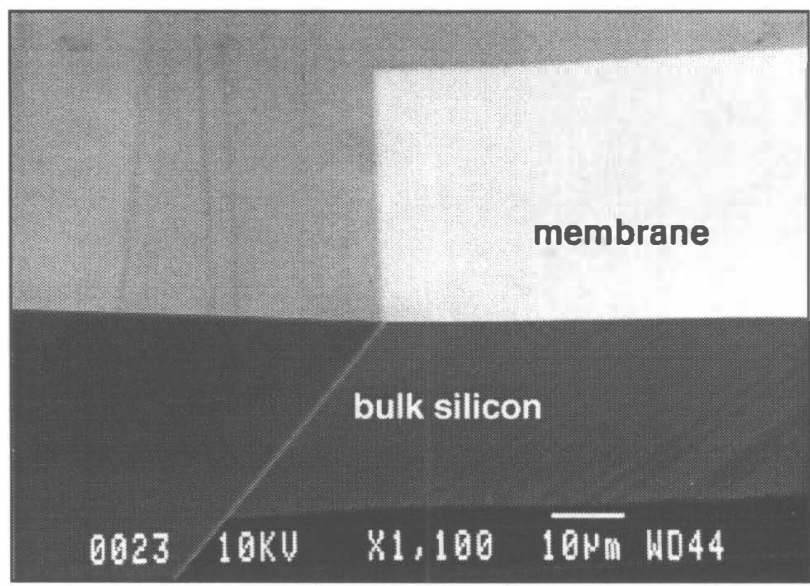

(a)

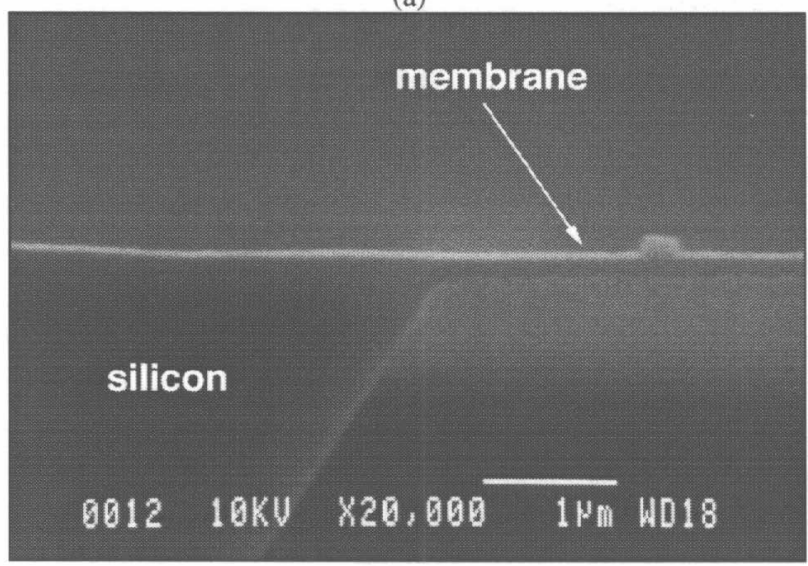

(b)

Figure 4: (a) SEM cross-section at one corner of the silicon membrane; (b) close-up view.

To fabricate the ultra-thin silicon bridge, the front side of the silicon wafer is implanted with boron at $40 \mathrm{keV}$ at a dose of $7 \times 10^{15} \mathrm{~cm}^{-2}$. After implantation, the wafer is patterned using RIE to etch through the implanted layer leaving behind only those regions that include the bridges and bonding anchors. The silicon wafer is then thinned down to $200 \mu \mathrm{m}$, and is subsequently bonded to a glass wafer. The glass substrate is patterned and recessed to a depth of $\sim 7 \mu \mathrm{m}$ using a mixture of $\mathrm{HF} / \mathrm{HNO}_{3}$ to create the bonding anchors. Glass processing is completed after patterning $\mathrm{Ti} / \mathrm{Pt} / \mathrm{Au}$ interconnect lines on it. Finally, the silicon wafer is electrostatically bonded to the glass wafer, and the sandwich is then immersed in EDP to dissolve away the undoped silicon, leaving the $\mathrm{p}^{++}$silicon structures mounted on the glass substrate. Figure 5 shows a thin $\mathrm{p}^{++}$silicon bridge bonded to glass. The thickness of the silicon bridge after EDP is about $2400 \AA$ and $2200 \AA$ for devices annealed at $1100^{\circ} \mathrm{C}$ for $30 \mathrm{sec}$. and for $15 \mathrm{sec}$., respectively. This measured thickness matches simulation results if one takes into account the finite etch rate of highly-doped silicon in EDP. Figure 6 shows the change in resistance of the bridge as a function of temperature. To eliminate the piezoresistive effect of silicon due to the thermal expansion of glass, the change of the resistance due to piezoresistive effect is calculated from:

$$
\frac{\Delta R}{R}=\frac{\Delta L}{L} G=\frac{\Delta L}{L} Y_{l} \pi_{l}
$$

where $\Delta L L$ is the strain of the resistor and $G$ the gauge factor which is equal to the product of Young's modulus and piezoresistive coefficient. The strain of the resistor is equal to the strain of the glass because the $\mathrm{p}^{++}$resistor is very thin and will not affect the overall strain. The thermal expansion coefficient of Corning 7740 glass is $32.5 \times 10^{-7} \mathrm{~cm} / \mathrm{cm} /{ }^{\circ} \mathrm{C}$ [6]. The Young's modulus of $\mathrm{p}^{++}$silicon is $\sim 175 \mathrm{GPa}$, and the piezoresistive coefficient is dominant by $\pi_{44} / 2$, which is $21.5 \times 10^{12}\left(\mathrm{~cm}^{2} / \mathrm{dyn}\right)$ [7]. As a result, $\Delta R / R$ is equal to $122 \mathrm{ppm} /{ }^{\prime} \mathrm{C}$, which is much smaller than the measured temperature coefficient of resistance (TCR) of the silicon bridges. Therefore, the resistance changes due to the piezoresistive effect can be neglected. The measured TCR varies from $1584 \mathrm{ppm} /{ }^{\circ} \mathrm{C}$ to $1864 \mathrm{ppm} /{ }^{\circ} \mathrm{C}$ based on six devices. These values closely match those reported by other researchers [8].

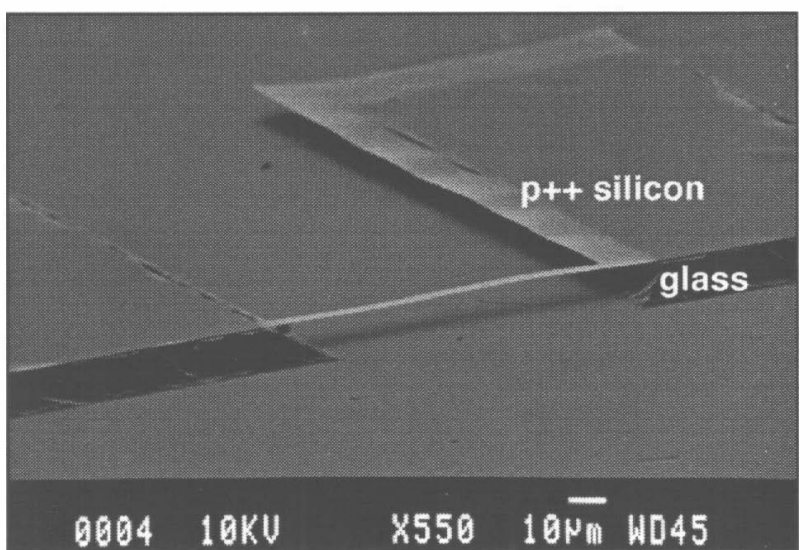

Figure 5: The $p^{++}$silicon bridge is $100 \mu m$ long, $5 \mu m$ wide, $0.24 \mu \mathrm{m}$ thick, and is bonded to glass anchors.

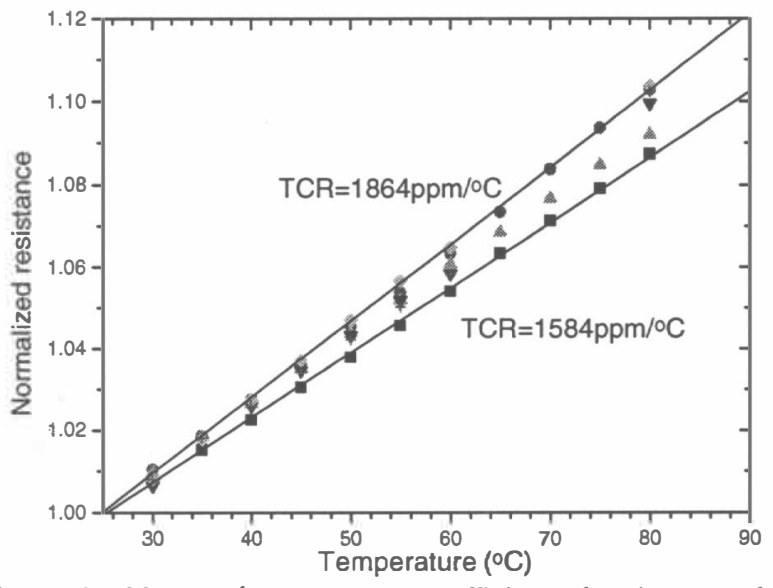

Figure 6: Measured temperature coefficient of resistance of $a$ $p^{++}$silicon resistor.

Combined with a dielectric diaphragm, the $\mathrm{p}^{++}$silicon resistors can be used in a piezoresistive sound detector fabricated using the dissolved wafer process [5]. Fabrication starts by recessing the silicon wafer with RIE to a depth of $1 \mu \mathrm{m}$, except in those areas that will later be bonded to the glass substrate. This recess is also used to create air ducts to equalize the pressure inside the cavity of the sound detector. Next a selective deep boron diffusion $(\sim 15 \mu \mathrm{m})$ is performed at $1175^{\circ} \mathrm{C}$ for 15 hours, which 
defines the rims of the sound detectors. A selective boron ion implantation at $40 \mathrm{keV}$ and a dose of $5 \times 10^{15} \mathrm{~cm}^{-2}$ is followed to define the piezoresistors. In order to activate the boron atoms, the wafer is annealed at $1000^{\circ} \mathrm{C}$ for $30 \mathrm{sec}$. After annealing, a $2260 \AA$ low temperature oxide layer is deposited at $420^{\circ} \mathrm{C}$ followed by the deposition of a $1900 \AA$ PECVD nitride layer to form the diaphragm. The initial stress of the diaphragm can be reduced by varying the relative thickness of the nitride and oxide films, thus improving the sensitivity. The wafer is metallized with a Ti/Pt layer to form lead transfers to metal lines on the glass substrate and is then thinned down to $\sim 100 \mu \mathrm{m}$. The glass wafer is patterned and recessed to a depth of $\sim 20 \mu \mathrm{m}$ to create the bonding anchors. This recess allows the formation of a large air gap under the silicon structure to reduce the possibility of clamping and the damping effect on the diaphragm. Glass processing is completed after patterning $\mathrm{Ti} / \mathrm{Pt} / \mathrm{Au}$ interconnect lines on it. Finally, the silicon wafer is electrostatically bonded to the glass wafer, and the sandwich is then immersed in EDP to dissolve away the undoped silicon, leaving the $\mathrm{p}^{+}$silicon devices mounted on the glass substrate.

Figure 7 shows the structure of this piezoresistive pressure sensor. It consists of a $0.4 \mu \mathrm{m}$-thick dielectric diaphragm suspended over a large air gap, and four $0.2 \mu \mathrm{m}$-thick $\mathrm{p}^{++}$ion-implanted piezoresistors. One of the advantage in using monocrystalline silicon instead of polysilicon is the high piezoresistive coefficient of single crystal silicon. The coefficient, $\pi_{44}$, is $43 \times 10^{12}\left(\mathrm{~cm}^{2} / \mathrm{dyn}\right)$ for $\mathrm{p}^{+}$-type monocrystalline silicon [7], which is $\sim 4$ times higher than that of p-type polysilicon piezoresistors [9]. Figure 8 shows the measured pressure sensitivity of the detector, which is $1.1 \mu \mathrm{V} / \mathrm{V} \cdot \mathrm{Pa}$, with a nonlinearity of $2 \%$ over the full scale range of $10 \mathrm{kPa}$.

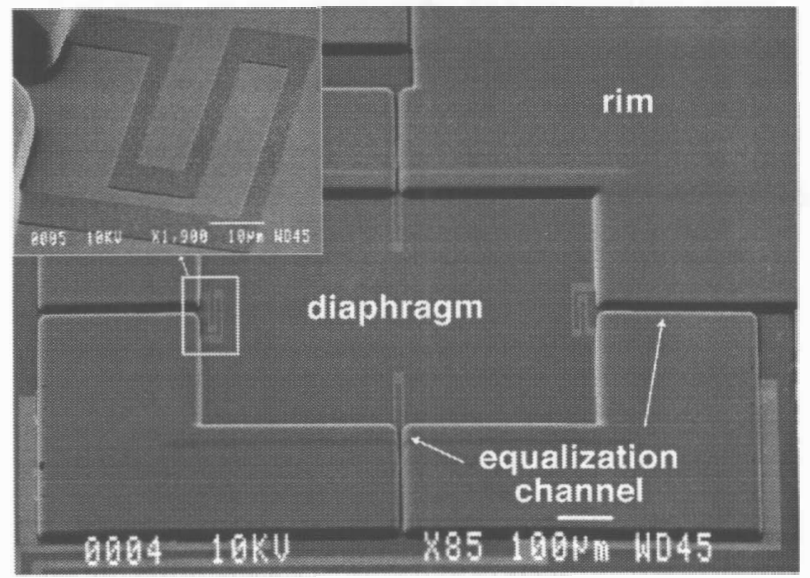

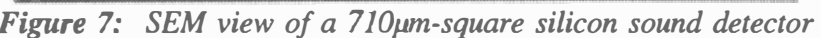
and a close-up view of one of the piezoresistors.

\section{CONCLUSIONS}

We have successfully utilized ion implantation, rapid thermal annealing, and EDP etch-stop to form submicron $\mathrm{p}^{\text {t+ }}$ microstructures. Boron implantation at an energy of $40 \mathrm{keV}$ and doses of $5 \times 10^{15} \mathrm{~cm}^{-2}$ and $7 \times 10^{15} \mathrm{~cm}^{-2}$ have been used. The thickness of the $\mathrm{p}^{+}$layer varies from 0.2 to $0.3 \mu \mathrm{m}$ depending on the annealing time and temperature. Since the highly-doped silicon layer is very thin, high temperature processes are eliminated to achieve a high boron concentration for an effective etch-stop. Boron ion implantation and etch-stop is a powerful technique because it allows the formation of a $\mathrm{p}^{++}$microstructure on a standard CMOS wafer since ion implantation and annealing can be easily performed at the end of the process. In addition, it is simply possible to use the resident $\mathrm{p}^{+}$layers in a standard CMOS process that are used for forming source/drain junctions of pMOS transistors. Finally, ion implantation provides flexibility in controlling the thickness and uniformity of the microstructure layer. We have reliably fabricated $\mathrm{p}^{+}$microstructures as thin as $0.2 \mu \mathrm{m}$.

\section{Acknowledgments}

The authors would like to thank Mr. Brendan Casey for wire bonding the devices. This work was sponsored by the Air Force Office of Scientific Research under contracts \#F49620-94-0184 and F49620-96-0293. The views and conclusions contained herein are those of the authors and should not be interpreted as necessarily representing the official policies or endorsements, either expressed or implied, of the Air Force Office of Science Research or the U.S. Government.

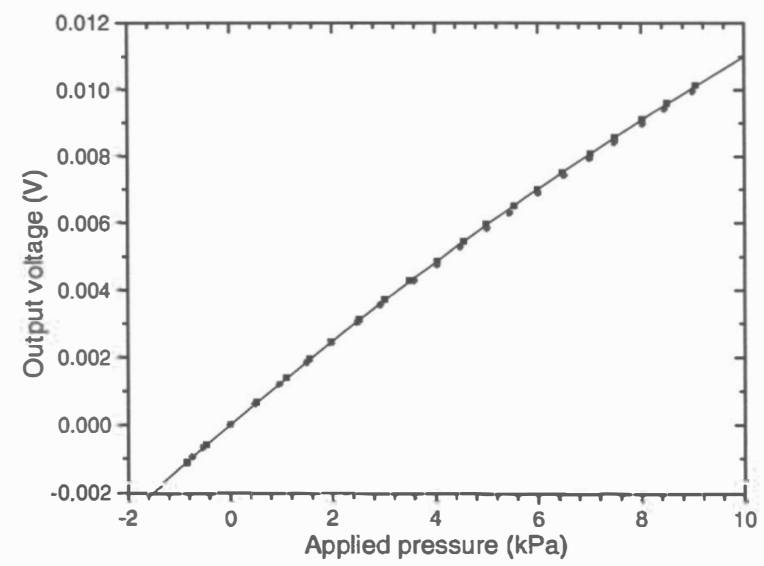

Figure 8: Measured pressure sensitivity of the sound detector is $1.1 \mu \mathrm{V} N \cdot \mathrm{Pa}$ with a nonlinearity of $2 \%$.

\section{REFERENCES}

1. A. Bohg, "Ethylene Diaminie-Pyrocatechol-Water Mixture Shows etching Anomaly in Boron-Doped Silicon", J. Electrochem. Soc., 118, 1971, pp.401-402.

2. Y. Gianchandani and K. Najafi, "A Bulk Silicon Dissolved Wafer Process for Microelectromechanical Devices", J-MEMS, Vol. 1, No. 2, 1992, pp. 77-85.

3. C. H. Mastrangelo, W. C. Tang, "Semiconductor Sensor Technologies", in Semiconductor Sensors, Ed. S. M. Sze, 1994.

4. B. Folkmer, P. Steiner, and W. Lang, "Silicon Nitride Membrane Sensors with Monocrystalline Transducers", Sensors and Actuators A 51, 1995, pp. 71-75.

5. C. Huang, K. Najafi, E. Alnajjar, C. Christophorou, A. Naguib, and H. Nagib, "Operation and Testing of Electrostatic Microactuators and Micromachined Sound Detectors for Active Control of High Speed Flows", Proc. Eleventh Annual Int. Workshop on MEMS, 1998, pp. 81-86.

6. ASM Engineering Materials Reference Book, Second Edition, 1994 , p. 320.

7. Y. Kanda, "Piezoresistance Effect of Silicon", Sensors and Actuators A 28, 1991, pp. 83-91.

8 P. W. Chapman, O. N. Tufte, J. D. Zook, and D. Long, "Electrical Properties of Heavily Doped Silicon", J. Applied Physics, V. 34, No. 11, 1963, pp.3291-3295.

9. H. Guckel, "Fine Grained Film and Its Application to Planar Pressure Transducers", Proc. $4^{\text {th }}$ Int. Conf. Solid-State Sensors and Actuators, Tokyo, Japan, 1987, pp. 277-282. 